\title{
Tratamiento con fosfato de sitagliptina en pacientes con hipoglucemia reactiva secundaria a disinsulinismo. Estudio controlado, aleatorizado y doble ciego
}

Daniel Cuevas-Ramos, Paloma Almeda-Valdés, Clara E. Meza-Arana, Griselda Brito-Córdova, Doris Georgina Ruiz-Gómez, Christian Razo y Francisco J. Gómez-Pérez

Departamento de Endocrinología y Metabolismo, Instituto Nacional de Ciencias Médicas y Nutrición Salvador Zubirán, Ciudad de México, México

\section{Resumen}

Objetivo: Evaluar el efecto de la sitagliptina en la corrección de la hipoglucemia reactiva sintomática. Métodos: Ensayo clínico, controlado con placebo, aleatorizado y doble ciego con 13 pacientes en el grupo de sitagliptina y 15 en el de placebo, de edad promedio ( \pm desviación estándar) 34.3 ( \pm 10.6) años e índice de masa corporal $24.6 \pm 5.1 \mathrm{~kg} / \mathrm{m}^{2}$, en su mayoría mujeres ( $n=24,85.7 \%$ ). Resultados: No se encontraron diferencias en el nivel basal de glucosa (92.3 \pm 18.9 vs. $93.4 \pm 17.3 \mathrm{mg} / \mathrm{dl} ; p=0.41)$ ni en la mediana [intervalo intercuartilar] de insulina (15.2 [8.8-43.7] vs. 14.7 $\mu \mathrm{U} / \mathrm{ml}$ [7.0-39.0]; $p=0.44$ ) entre el grupo de placebo y el de sitagliptina, respectivamente. El grupo con sitagliptina recuperó la fase temprana de secreción de insulina. Esto se asoció con un valor más alto de glucosa en la fase tardía (79.5 [74.2-83.0] vs. 83.5 [81.289.7]; $p=0.003$ ) y con un valor de insulina semejante (13.0 [5.0-34.4] vs. 13.8 [4.8-30.6]; $p=0.32$ ). La frecuencia de síntomas fue significativamente menor en el grupo con sitagliptina $(p<0.0001)$. Conclusiones: La sitagliptina mejoró la fase temprana de secreción de insulina y redujo la sintomatología postprandial, lo que sugiere un nuevo tratamiento para la hipoglucemia reactiva por disinsulinismo.

PALABRAS CLAVE: Hipoglucemia reactiva. Insulina. Glucosa. Diabetes.

\begin{abstract}
Objective: We evaluated the effect of sitagliptin in correction of symptomatic reactive hypoglycemia. Methods: Randomized, double blind, placebo-controlled clinical trial. Thirteen patients treated with sitagliptin and 15 with placebo, with mean age of $34.3 \pm 10.6$ years and body mass index of $24.6 \pm 5.1 \mathrm{~kg} / \mathrm{m}^{2}$, mostly women ( $\left.n=24,85.7 \%\right)$, were evaluated. Results: Basal glycemia was similar in placebo versus sitagliptin (92.3 $\pm 18.9 \mathrm{vs} .93 .4 \pm 17.3 \mathrm{mg} / \mathrm{dl} ; p=0.41)$ as was median and interquartile range of insulin (15.2 [8.8-43.7] vs. $14.7 \mu \mathrm{U} / \mathrm{ml}$ [7.0-39.0]; $p=0.44)$. Patients with sitagliptin recovered the first phase insulin secretion (FISP). This was related with higher glucose level at the end of the oral glucose tolerance test (79.5 [74.2-83.0] vs. 83.5 [81.2-89.7]; $p=0.003)$ with a similar insulin median level (13.0 [5.0-34.4] vs. 13.8 [4.8-30.6]; $p=0.32)$. Symptomatology was significantly lower under sitagliptin treatment $(p<0.0001)$. Conclusions: Sitagliptin improved FISP and reduced post-prandial symptomatology. These results suggest a novel therapeutic option for patients with reactive hypoglycemia related with FISP.
\end{abstract}

KEY WORDS: Reactive hypoglycemia. Insulin. Glucose. Diabetes.

\section{Correspondencia:}

Francisco J. Gómez-Pérez

Vasco de Quiroga, 15

Col. Belisario Domínguez Sección XVI, Del. Tlalpan Fecha de recepción: 07-08-2017

C.P. 14080 , Ciudad de México, México

Fecha de aceptación: 07-09-2017

E-mail: fcojgomezp@aol.com

DOI://dx.doi.org/10.24875/GMM.M17000007

Gac Med Mex. 2017;153;Sup 2:S51-S59

Contents available at PubMed www.anmm.org.mx 


\section{Introducción}

La sitagliptina es un inhibidor de la enzima dipeptidil peptidasa tipo 4 (DPP-4) que representa un nuevo abordaje terapéutico para el tratamiento de diabetes tipo $2^{1}$. Este agente actúa al inhibir la enzima DPP-4, que se encarga de degradar las hormonas incretinas, como son el polipéptido insulinotrópico dependiente de glucosa (GIP, glucose-dependent insulinotropic polypeptide) $)^{2-4}$ y el péptido similar al glucagón tipo 1 (GLP-1, glucagon-like peptide 1) ${ }^{2-68}$, estabilizando los niveles de la forma intacta (activa) de las hormonas incretinas ${ }^{7}$. El GLP-1 activo y el GIP estimulan la biosíntesis y la liberación de insulina dependiente de glucosa, y el GLP-1 también suprime la liberación de glucagón, retrasa el vaciamiento gástrico y aumenta la saciedad ${ }^{8}$. Las concentraciones circulantes de GLP-1 y GIP disminuyen rápido por la inactivación enzimática llevada a cabo, principalmente, por la DPP-4, así como por eliminación renal ${ }^{9,10}$. Al inhibir la DPP-4, el fosfato de sitagliptina evita el metabolismo y la eliminación de las principales incretinas reconocidas hasta el momento, ocasionando mayores concentraciones séricas de GLP-1 y GIP. En estudios clínicos con los inhibidores de DPP-4 se ha logrado demostrar el efecto agonista particularmente de GLP 1, incluyendo la estimulación de la secreción de insulina, y la inhibición de la secreción de glucagón y en estudios in vitro preservación de las células beta en el páncreas, al estimular la proliferación celular y causar una inhibición de la apoptosis ${ }^{9-11}$. El perfil de eventos adversos es equivalente al de un placebo y no se ha relacionado con cambios de peso (cambio promedio de $0.7 \mathrm{~kg}$ con sitagliptina y $0.6 \mathrm{~kg}$ con placebo) en dos ensayos controlados durante 24 semanas de tratamiento ${ }^{12-16}$. La dosis recomendada es de $100 \mathrm{mg}$ al día, con o sin alimento.

La hipoglucemia reactiva se refiere al descenso de la glucosa después de los alimentos. Comúnmente, los individuos con este tipo de hipoglucemia tienen una personalidad característica con hipersomatización e hipocondría ${ }^{17}$. Aunque cualquier causa de hipoglucemia de ayuno puede ocasionar hipoglucemia posprandial, como por ejemplo insulinomas, hipopituitarismo, alcohol, ingestión de sulfonilureas, hipotiroidismo, deficiencia de hormona del crecimiento o deficiencia de cortisol, cinco son las causas relacionadas con hipoglucemia posprandial: la alimentaria, la prediabetes, la idiopática, la iatrogénica y la hipoglucemia funcional ${ }^{18,19}$. De estos subtipos, la hipoglucemia de interés en este estudio es la ocasionada en los pacientes con alteración en la tolerancia a la glucosa o prediabetes. Estos casos tienen retraso en la liberación de insulina, que produce supresión de la liberación endógena de glucosa y disminuye la eficiencia temprana de capturar la glucosa por los tejidos, ocasionando hiperglucemia con hiperinsulinemia tardía ${ }^{20-21}$. El exceso de insulina está en desequilibrio con los valores de glucosa, produciendo hipoglucemia a las 3 a 5 horas, lo cual puede hacerse evidente durante la curva de tolerancia a la glucosa oral (CTOG). Casi siempre el descenso de la glucosa es leve y el tratamiento va dirigido a mejorar la tolerancia a los hidratos de carbono, como disminuir de peso en caso de obesidad, uso de metformina o inhibidores de la alfa glucosidasa, así como prevención en la dieta al restringir los alimentos altos en hidratos de carbono, con porciones pequeñas de alimentos, sin azúcares simples, y aumentar la fibra. La respuesta al tratamiento es variable y en algunos pacientes la sintomatología persiste, pues al no corregirse el patrón de secreción de insulina, el problema recurre si no se tiene buen apego a las indicaciones descri$\operatorname{tas}^{18,20,21}$. Usualmente estos pacientes tienen familiares enfermos de diabetes mellitus tipo 2 y datos de resistencia a la insulina, como acantosis nigricans y acrocordones en el cuello. Como la ausencia de estos datos clínicos no lo descarta, el diagnóstico se funda en el automonitoreo de la glucosa durante la sintomatología en el periodo posprandial. La finalidad es demostrar la tríada de Whipple, la cual consiste en la detección de unos valores de glucosa menores de $50 \mathrm{mg} / \mathrm{dl}$ en presencia de síntomas, los cuales se corrigen administrando algún tipo de alimento. Si la hipoglucemia se confirma, es recomendable realizar una CTOG para determinar el grado de hiperglucemia, hiperinsulinemia y el momento de la hipoglucemia, para después administrar el tratamiento adecuado 17,18,20-22. En este estudio se investigó si la administración de sitagliptina mejora la fase temprana de secreción de insulina y corrige la hipoglucemia reactiva postprandial con mejoría en la sintomatología.

\section{Métodos}

Se trata de un ensayo clínico aleatorizado, doble ciego y controlado con placebo sobre el papel de la administración de fosfato de sitagliptina (Januvia $®$ ) en pacientes con hipoglucemia reactiva. 


\section{Evaluaciones}

Los sujetos fueron seleccionados de la consulta externa de Endocrinología del Instituto Nacional de Ciencias Médicas y Nutrición Salvador Zubirán, y los casos incluidos en el estudio fueron evaluados con historia clínica y exploración física completa con el fin de confirmar el cumplimiento de los criterios de inclusión y descartar la presencia de criterios de exclusión. Además, se realizó una evaluación nutricional mediante recordatorios de dieta de 24 horas. Se evaluó el contenido energético de la dieta isocalórica recomendada, así como los constituyentes de la misma, es decir, proteínas, hidratos de carbono, lípidos, vitaminas y minerales, de acuerdo con las tablas de contenido de nutrimentos de los alimentos mexicanos ${ }^{23}$. Por otro lado, se hizo una evaluación de las medidas antropométricas, circunferencia de cintura y cadera, talla, peso e índice de masa corporal (IMC). También se realizó una evaluación de laboratorio, previo ayuno de 12 horas, que consistió en la realización de CTOG de 5 horas, utilizando 75 gramos de glucosa, con medición de glucosa e insulina a los $0,5,10,15,20$, $25,30,60,90,120,180,240$ y 300 minutos. En las mujeres que se encontraban en posibilidades de embarazo se realizó la determinación de la fracción beta de la gonadotropina coriónica para descartarlo.

\section{Asignación de tratamiento y cegamiento}

La asignación del tratamiento fue al azar. El mecanismo de aleatorización fue con el uso de sobres cerrados utilizando bloques de cuatro. Cabe mencionar que personal ajeno al estudio se encargó de entregar a cada paciente el tratamiento completo de acuerdo con el orden establecido. El grupo experimental consistió en 13 sujetos con diagnóstico de hipoglucemia reactiva por alteración en la tolerancia a la glucosa o prediabetes. El segundo grupo consistió en 15 sujetos control sin hipoglucemia reactiva. La duración de la fase de estudio en los 28 pacientes fue de 4 semanas; inicialmente, 2 semanas de preparación para ingresar al estudio siguiendo una dieta isocalórica por 15 días antes de iniciar el tratamiento, y después continuaron con el tratamiento asignado por 2 semanas. Se realizó una evaluación inicial antes del tratamiento y al final del mismo. En ambos momentos se llevaron a cabo las evaluaciones clínica y de laboratorio con la finalidad de detectar alguna alteración que pudiera haberse presentado por motivo del tratamiento asignado, así como una evaluación nutricional para establecer que no hubieran ocurrido cambios en las variables antropométricas o en el peso. Se evaluó el apego al tratamiento mediante el sistema de conteo de pastillas y cuestionario directo al paciente. El cegamiento se logró con el uso de sobres cerrados, y el conteo de tabletas fue realizado por un investigador que no realizó ninguna otra evaluación. Dos colaboradores implementaron los cuestionarios de síntomas y estaban cegados para el tratamiento de los pacientes. El personal de enfermería que tomó las muestras también desconocía el tipo de tratamiento. Los pacientes estuvieron cegados al tipo de tratamiento que estaban recibiendo.

\section{Definición de hipoglucemia reactiva y disinsulinismo}

Se utilizó la siguiente definición de hipoglucemia reactiva: la presencia de glucosa capilar $<65 \mathrm{mg} / \mathrm{dl}$ en las siguientes 2 a 4 horas después de consumir alimentos, asociada con síntomas adrenérgicos. El disinsulinismo se definió como el retraso en la secreción de la fase temprana de insulina con un pico de secreción después de los 30 minutos y ausencia de retornar a su nivel basal en la tercera hora de la CTOG $^{17-22}$. Se utilizaron los criterios estándar de prediabetes de la Asociación Americana de Diabetes de 2011, actuales hasta la fecha, definida como glucosa de ayuno $\geq 100 \mathrm{mg} / \mathrm{dl}$ o glucosa a las 2 horas de la CTOG entre 140 y $199 \mathrm{mg} / \mathrm{dl}^{24}$.

\section{Evaluación de los síntomas}

Los síntomas se evaluaron utilizando una escala visual análoga. Se solicitó a los pacientes que marcaran con una equis $(x)$ la intensidad con que percibieron cada molestia o síntoma en el momento de notar la hipoglucemia postprandial. La escala es del 1 al 10, en la que el 1 representa una sensación muy leve y el 10 una sensación muy intensa. Se les indicó elegir un solo número para cada síntoma (Fig. 1).

\section{Sujetos estudiados}

Se estudiaron sujetos con edad entre 18 y 45 años, de ambos sexos, con IMC entre 25 y $30 \mathrm{~kg} / \mathrm{m}^{2}$, y diagnóstico confirmado de hipoglucemia reactiva por alteración en la tolerancia a la glucosa o prediabetes y disinsulinismo mediante la CTOG basal realizada con fines de este estudio. Se excluyeron aquellos 


\begin{tabular}{|c|c|c|c|c|c|c|c|c|c|c|}
\hline Síntomas & 1 & 2 & 3 & 4 & 5 & 6 & 7 & 8 & 9 & 10 \\
\hline \multicolumn{11}{|l|}{ Ansiedad } \\
\hline \multicolumn{11}{|l|}{ Palpitaciones } \\
\hline \multicolumn{11}{|l|}{ Irritabilidad } \\
\hline \multicolumn{11}{|l|}{ Temblor } \\
\hline \multicolumn{11}{|l|}{ Diaforesis } \\
\hline \multicolumn{11}{|l|}{ Hambre } \\
\hline \multicolumn{11}{|l|}{ Mareo } \\
\hline \multicolumn{11}{|l|}{ Hormigueo } \\
\hline \multicolumn{11}{|l|}{$\begin{array}{l}\text { Visión } \\
\text { borrosa }\end{array}$} \\
\hline \multicolumn{11}{|l|}{$\begin{array}{l}\text { Dificultad } \\
\text { para } \\
\text { concentrarse }\end{array}$} \\
\hline \multicolumn{11}{|l|}{ Debilidad } \\
\hline \multicolumn{11}{|l|}{ Somnolencia } \\
\hline $\begin{array}{l}\text { Las definiciones o } \\
\text { aprehensión o mie } \\
\text { sensación de enoj } \\
\text { rítmico, usualment } \\
\text { comer; mareo: sen } \\
\text { piquetitos o adorm } \\
\text { observar las cosas } \\
\text { actividades; debill } \\
\text { de quererse dorm }\end{array}$ & $\begin{array}{l}\text { ión d } \\
\text { mient } \\
n \text { tod } \\
\text { : sens }\end{array}$ & & ool & trem & 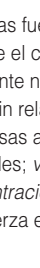 & tr & $\begin{array}{l}\text { tividac } \\
\text { nueve } \\
\text { : la vis } \\
\text { para } \\
\text { iernas }\end{array}$ & $\begin{array}{l}\text { ica; } h \\
\text { ormic } \\
\text { se nu } \\
\text { ocarse } \\
\text { somn }\end{array}$ & $\begin{array}{l}\text { ngust } \\
\text {; irrita } \\
\text { nto in } \\
\text { bre: d } \\
\text { o: sen } \\
\text { y no } \\
\text { realiz } \\
\text { cia: }\end{array}$ & $\begin{array}{l}\text { dad: } \\
\text { untario, } \\
\text { os de } \\
\text { ilón de } \\
\text { oosible } \\
\text { as } \\
\text { ación }\end{array}$ \\
\hline
\end{tabular}

Figura 1. Escala visual analógica para la evaluación de la intensidad de la sintomatología.

sujetos con función renal anormal, definida por creatinina $>1.2 \mathrm{mg} / \mathrm{dl}$ y depuración de creatinina $<80 \mathrm{ml} / \mathrm{min}$, con algún otro tipo de hipoglucemia reactiva (alimentaria, idiopática, iatrogénica o funcional), alguna enfermedad aguda al momento de la evaluación inicial, enfermedad grave u hospitalización en los 6 meses previos al ingreso al estudio, evidencia de enfermedad mortal a corto plazo, toma de medicamentos que pudieran interferir con el tratamiento administrado en el estudio o con las valoraciones de laboratorio del mismo, con contraindicación para el uso de fosfato de sitagliptina (p. ej., pacientes con diabetes tipo 1 o con algún tipo de nefropatía), con incapacidad para usar la vía oral, con incapacidad para entender las indicaciones, con intolerancia conocida a las sustancias que serán utilizadas en el estudio, y mujeres con intención de embarazo durante el periodo de estudio o con embarazo actual. Se planeó eliminar los casos que no cumplieran con el 100\% del tratamiento asignado, con presencia de intolerancia o efectos adversos con el tratamiento administrado, alguna enfermedad aguda durante el periodo de estudio y aquellos que no desearan continuar participando, presencia de un incremento en la frecuencia de hipoglucemias con cualquiera de las maniobras, apego inferior al $90 \%$ en la dieta isocalórica, o prueba de embarazo positiva durante el periodo de estudio. Sin embargo, ningún caso presentó dichas características y no hubo eliminaciones.

\section{Frecuencia de las mediciones de laboratorio}

Las mediciones se realizaron al inicio del estudio, que se consideraron mediciones basales, y después de haber recibido 2 semanas de tratamiento. El laboratorio del Departamento de Endocrinología y Metabolismo del Instituto Nacional de Ciencias Médicas y Nutrición Salvador Zubirán realizó todas las mediciones bioquímicas mediante procedimientos estandarizados. Las mediciones se realizaron con ensayos comerciales. Todos los sujetos se citaron después de un ayuno de 8 a 12 horas para las determinaciones bioquímicas. La glucosa, el colesterol total y triglicéridos se determinaron utilizando métodos enzimáticos (Boehringer Mannheim). Los valores del colesterol ligado a lipoproteínas de alta se cuantificaron después de 
precipitación con ácido fosfotúngstico y $\mathrm{Mg}^{2+}$ (Boehringer Mannheim). El colesterol ligado a lipoproteínas de baja densidad se estimó utilizando la fórmula de Friedewal. La insulina plasmática fue determinada con un ensayo inmunoenzimático en micropartículas (MEIA, Abbott Laboratories). La creatinina fue cuantificada con ensayos colorimétricos comerciales (Beckman Coulter, Inc). Todo el equipo se calibró con regularidad utilizando las muestras de referencia proporcionadas por el fabricante.

\section{Análisis estadístico}

Utilizando como desenlace primario el cambio en las cifras de insulina $(\mu \mathrm{U} / \mathrm{ml})$ en $5 \mu \mathrm{U} / \mathrm{ml}$ a los $30 \mathrm{mi}-$ nutos de secreción (fase temprana), con un valor de alfa a dos colas de 0.05 , con una beta de 0.20 (poder del $80 \%$ ) y una desviación estándar de $4 \mu \mathrm{U} / \mathrm{ml}$, se realizó un cálculo de tamaño de muestra de 10 pacientes por grupo. Se agregó un $20 \%$ por pérdidas anticipadas, lo que resultó en un total de 24 pacientes $(\mathrm{N}=2(16)(7.84) / 25=10$ por grupo $=20+4=$ 24). Se realizó estadística descriptiva de todas las variables y se reportaron como media y desviación estándar aquellas con distribución normal, y como mediana e intervalo intercuartilar aquellas con distribución sesgada. Las variables entre grupos se compararon con prueba t de Student o $U$ de Mann Whitney, según correspondiera. Las variables categóricas se compararon con la prueba de ji al cuadrado de
Pearson o la prueba exacta de Fisher. En caso de comparaciones pareadas se utilizaron las pruebas de $t$ de Student para muestras pareadas, Wilcoxon o McNemar, dependiendo del tipo de variables y su distribución. Se calculó el área bajo la curva (AUC) utilizando la fórmula de suma de los trapezoides. La fase temprana se definió del minuto 0 al 30 de la CTOG, y la fase tardía del 60 al 300. Se consideró significativo un valor de $p<0.05$ con pruebas a dos colas. Se utilizó el programa de estadística SPSS 17.0 (Chicago, IL).

\section{Resultados}

Se incluyeron 13 sujetos en el grupo de sitagliptina y 15 en el grupo de placebo, de los cuales el $85.7 \%$ fueron mujeres $(n=24)$. El promedio $( \pm$ desviación estándar [DE]) de edad fue de $34.3 \pm 10.6$ años, y el IMC fue de $24.6 \pm 5.1 \mathrm{~kg} / \mathrm{m}^{2}$. Las características basales, junto con los valores de glucosa e insulina durante la CTOG, estratificadas por grupos, se muestran en la tabla 1.

No se encontraron diferencias entre la glucosa basal $(81.6 \pm 7.7$ vs. $81.2 \pm 7.2 \mathrm{mg} / \mathrm{dl} ; p=0.21)$ ni en la mediana (intervalo intercuartilar) del valor de la insulina [6.3 (5.1-8.8)] vs. $6.4(5.3-10.3) \mu \mathrm{U} / \mathrm{ml} ; \mathrm{p}=0.80]$ entre los grupos de sitagliptina y control, respectivamente. Tampoco se encontraron diferencias basales en el AUC en los valores de glucosa e insulina durante la CTOG (Tabla 2).

Tabla 1. Características clínicas y bioquímicas de la población estudiada antes y después de recibir 2 semanas de tratamiento con sitagliptina o placebo $(n=28)$

\begin{tabular}{|c|c|c|c|c|c|c|}
\hline \multirow[t]{2}{*}{ Variables } & \multicolumn{2}{|c|}{ Antes } & \multirow[t]{2}{*}{$p$} & \multicolumn{2}{|c|}{ Después } & \multirow[t]{2}{*}{$p$} \\
\hline & Sitagliptina & Placebo & & Sitagliptina & Placebo & \\
\hline Edad (años) & $31.8 \pm 7.9$ & $37.1 \pm 12.9$ & 0.21 & - & - & - \\
\hline IMC $\left(\mathrm{kg} / \mathrm{m}^{2}\right)$ & $25.9 \pm 6.0$ & $23.2 \pm 3.3$ & 0.14 & $25.2 \pm 5.5$ & $23.2 \pm 3.1$ & 0.14 \\
\hline $\mathrm{CC}(\mathrm{cm})$ & $87.1 \pm 14.7$ & $78.6 \pm 7.5$ & 0.06 & $85.9 \pm 14.6$ & $75.7 \pm 7.3$ & 0.11 \\
\hline CCad (cm) & $103.1 \pm 13.9$ & $96.5 \pm 6.7$ & 0.11 & $102.9 \pm 12.5$ & $95.8 \pm 4.7$ & 0.19 \\
\hline ICC & $0.84 \pm 0.06$ & $0.81 \pm 0.04$ & 0.21 & $0.83 \pm 0.05$ & $0.80 \pm 0.01$ & 0.19 \\
\hline TAS (mmHg) & $104.5 \pm 10.8$ & $110.0 \pm 17.5$ & 0.36 & $104.2 \pm 11.8$ & $110.2 \pm 15.5$ & 0.39 \\
\hline TAD (mmHg) & $69.7 \pm 6.0$ & $70.8 \pm 7.9$ & 0.68 & $70.1 \pm 5.8$ & $71.5 \pm 5.5$ & 0.41 \\
\hline Glucosa basal & $81.6 \pm 7.7$ & $81.2 \pm 7.2$ & 0.90 & $81.9 \pm 8.7$ & $82.7 \pm 5.5$ & 0.77 \\
\hline Glucosa 2.5' & $81.1 \pm 7.8$ & $81.4 \pm 6.5$ & 0.92 & $81.4 \pm 8.8$ & $83.1 \pm 4.7$ & 0.53 \\
\hline Glucosa 5' & $81.2 \pm 7.9$ & $82.7 \pm 83.0$ & 0.61 & $79.0 \pm 8.3$ & $83.1 \pm 6.1$ & 0.44 \\
\hline Glucosa 7.5' & $81.3 \pm 8.2$ & $83.4 \pm 7.0$ & 0.46 & $80.4 \pm 8.4$ & $84.8 \pm 6.1$ & 0.39 \\
\hline
\end{tabular}


Tabla 1. Características clínicas y bioquímicas de la población estudiada antes y después de recibir 2 semanas de tratamiento con sitagliptina o placebo $(n=28)$ (Continued)

\begin{tabular}{|c|c|c|c|c|c|c|}
\hline \multirow[t]{2}{*}{ Variables } & \multicolumn{2}{|c|}{ Antes } & \multirow[t]{2}{*}{$p$} & \multicolumn{2}{|c|}{ Después } & \multirow[t]{2}{*}{$p$} \\
\hline & Sitagliptina & Placebo & & Sitagliptina & Placebo & \\
\hline Glucosa 10' & $83.2 \pm 8.9$ & $88.2 \pm 8.2$ & 0.13 & $82.0 \pm 9.4$ & $90.1 \pm 7.7$ & 0.07 \\
\hline Glucosa 15' & $95.0 \pm 13.4$ & $98.1 \pm 13.5$ & 0.54 & $92.9 \pm 14.0$ & $102.6 \pm 10.9$ & 0.04 \\
\hline Glucosa 20' & $110.5 \pm 15.9$ & $114.9 \pm 15.9$ & 0.47 & $104.1 \pm 20.6$ & $116.4 \pm 13.4$ & 0.07 \\
\hline Glucosa 25' & $123.8 \pm 20.0$ & $124 \pm 18.9$ & 0.95 & $114.5 \pm 28.5$ & $126.0 \pm 17.0$ & 0.19 \\
\hline Glucosa 30' & $122.3 \pm 25.8$ & $128 \pm 21.9$ & 0.49 & $118.5 \pm 34.9$ & $129.2 \pm 19.1$ & 0.33 \\
\hline Glucosa 60' & $92.3 \pm 37.7$ & $98.1 \pm 26.1$ & 0.64 & $90.3 \pm 30.9$ & $107.1 \pm 33.0$ & 0.18 \\
\hline Glucosa 90' & $82.7 \pm 30.9$ & $80.2 \pm 19.1$ & 0.79 & $83.0 \pm 32.0$ & $84.6 \pm 70.0$ & 0.87 \\
\hline Glucosa 120' & $80.3 \pm 20.0$ & $73.0 \pm 11.1$ & 0.24 & $76.4 \pm 21.4$ & $79.6 \pm 17.7$ & 0.67 \\
\hline Glucosa 180' & $74.4 \pm 13.9$ & $73.7 \pm 10.7$ & 0.87 & $79.6 \pm 12.4$ & $75.2 \pm 8.7$ & 0.29 \\
\hline Glucosa 240' & $77.5 \pm 9.5$ & $78.0 \pm 6.5$ & 0.88 & $79.5 \pm 7.6$ & $79.8 \pm 5.1$ & 0.91 \\
\hline Glucosa 300' & $82.0 \pm 7.9$ & $83.5 \pm 4.6$ & 0.53 & $83.5 \pm 6.7$ & $81.2 \pm 4.1$ & 0.26 \\
\hline Insulina 0' & $6.3(5.1-8.8)$ & $6.4(5.3-10.3)$ & 0.80 & $5.3(4.0-8.4)$ & $7.4(5.3-13.3)$ & 0.06 \\
\hline Insulina 2.5' & $6.5(5.0-10.3)$ & $7.0(5.9-10.4)$ & 0.32 & $5.8(4.5-9.8)$ & $8.7(6.9-18.0)$ & 0.21 \\
\hline Insulina 5' & $7.0(5.3-12.8)$ & $9.3(7.7-12.1)$ & 0.26 & $6.9(5.2-11.2)$ & $9.5(7.2-18.8)$ & 0.09 \\
\hline Insulina 7.5' & $7.4(5.8-12.7)$ & $11.0(6.6-15.9)$ & 0.23 & $8.2(4.8-11.8)$ & $9.9(7.6-24.1)$ & 0.15 \\
\hline Insulina 10' & $10.5(6.6-16.7)$ & $12.9(10.5-21.8)$ & 0.18 & $8.3(5.7-19.1)$ & $13.8(8.3-31.6)$ & 0.09 \\
\hline Insulina $15^{\prime}$ & $21.8(13.9-39)$ & $23.2(14.7-30.5)$ & 0.83 & $20.2(14.3-41.7)$ & $27.9(17.6-61.7)$ & 0.26 \\
\hline Insulina 20' & $43.2(29.4-64.7)$ & $37.5(34.0-64.3)$ & 0.90 & $47.3(25.7-63.2)$ & $45.8(28.9-125.8)$ & 0.36 \\
\hline Insulina 25' & $59.9(39.6-91.0)$ & $64.3(45.1-89.1)$ & 0.90 & $64.0(36.5-82.9)$ & $61.6(37.1-148.2)$ & 0.71 \\
\hline Insulina 30' & $43.2(29.4-64.7)$ & 70.8 (47.2-107.5) & 0.25 & $57.2(45.4-89.5)$ & $67.3(41.4-150.3)$ & 0.76 \\
\hline Insulina 60' & $36.5(19.9-65.2)$ & $48.9(39.1-77.5)$ & 0.07 & $38.3(22.3-60.1)$ & $67.6(37.0-78.8)$ & 0.04 \\
\hline Insulina 90' & $26.4(14.5-65.8)$ & $33.5(23.6-67.6)$ & 0.40 & $31.3(19.2-48.3)$ & $34.8(22.5-45.3)$ & 0.94 \\
\hline Insulina 120' & $20.4(11.0-30.0)$ & $18.2(9.8-30.2)$ & 0.85 & $18.2(7.9-22.8)$ & $16.6(12.2-23.3)$ & 0.50 \\
\hline Insulina 180' & $7.0(5.0-14.0)$ & $6.0(4.9-15.3)$ & 0.87 & $8.5(4.5-12.9)$ & $9.5(5.5-12.9)$ & 0.59 \\
\hline Insulina 240' & $4.8(3.4-7.5)$ & $4.6(3.8-7.1)$ & 0.64 & $4.6(3.3-6.9)$ & $4.7(3.5-11.4)$ & 0.56 \\
\hline Insulina 300' & $4.3(3.6-7.3)$ & $5.0(4.1-7.1)$ & 0.38 & $3.8(3.0-5.4)$ & $5.3(3.7-8.0)$ & 0.11 \\
\hline
\end{tabular}

Los datos se expresan como promedio \pm desviación estándar o mediana (intervalo intercuartilar).

CC: circunferencia de la cintura; CCad: circunferencia de la cadera; IMC: índice de masa corporal; PAD: presión arterial diastólica; PAS: presión arterial sistólica.

Efecto de sitagliptina en la fase temprana y tardía de la CTOG

El AUC de la fase de secreción temprana de glucosa (minutos 0 a 30) mostró una reducción significativa (10263 [9380-10177] vs. 11117 [10286-11255]; $p=0.01$ ) en el grupo de sitagliptina en comparación con el grupo control, respectivamente (Tabla 2). El promedio $( \pm \mathrm{DE})$ de los valores de glucosa en la fase de secreción temprana también mostraron diferencia significativa, siendo menores en el grupo de sitagliptina (93.4 \pm 4.9$)$ que en el grupo control (99.8 \pm 8.3; $p=0.02$ ) (Tabla 2). Este cambio estuvo asociado con un incremento del AUC de la fase de secreción temprana de insulina (2728 [1559-3025] vs. 2106 [12112951]; $p=0.01$ ) (Tabla 2). La mediana (intervalo intercuartilar) de la insulina en la fase temprana fue mayor en el grupo de sitagliptina (13.8 [10.8-31.9]) que 
Tabla 2. Áreas bajo la curva y valores absolutos de glucosa e insulina antes y después de 2 semanas de sitagliptina o placebo $(n=28)$

\begin{tabular}{|c|c|c|c|c|c|c|}
\hline & \multicolumn{2}{|c|}{ Antes } & \multirow[t]{2}{*}{$p$} & \multicolumn{2}{|c|}{ Después } & \multirow[t]{2}{*}{$p$} \\
\hline & Sitagliptina & Placebo & & Sitagliptina & Placebo & \\
\hline $\begin{array}{l}\text { AUC } \\
\text { Glu (0-300') }\end{array}$ & $\begin{array}{c}91,561 \\
(82,260- \\
116,965)\end{array}$ & $\begin{array}{c}100,035 \\
(95,983- \\
108,923)\end{array}$ & 0.83 & $\begin{array}{r}94,207 \\
(82,835- \\
115,938)\end{array}$ & $\begin{array}{c}101,147 \\
(93,858- \\
118,994)\end{array}$ & 0.47 \\
\hline Ins (0-300') & $\begin{array}{c}21,564 \\
(30,240-44,712)\end{array}$ & $\begin{array}{c}33,355 \\
(23,766-51,650)\end{array}$ & 0.47 & $\begin{array}{c}27,883 \\
(22,420-43,760)\end{array}$ & $\begin{array}{c}35,465 \\
(24,961-59,818)\end{array}$ & 0.34 \\
\hline F Temp Glu & $\begin{array}{c}10,383 \\
(9312-11,702)\end{array}$ & $\begin{array}{c}10,727 \\
(10,004-11,618)\end{array}$ & 0.56 & $\begin{array}{c}10,263 \\
(9380-10,177)\end{array}$ & $\begin{array}{c}11,117 \\
(10,286-11,255)\end{array}$ & 0.01 \\
\hline F Temp Ins & $\begin{array}{c}2673 \\
(1512-3640)\end{array}$ & $\begin{array}{c}2131 \\
(2002-3266)\end{array}$ & 0.56 & $\begin{array}{c}2728 \\
(1559-3025)\end{array}$ & $\begin{array}{c}2106 \\
(1211-2951)\end{array}$ & 0.01 \\
\hline F Tardía Glu & $\begin{array}{c}81,000 \\
(72,652-105,060)\end{array}$ & $\begin{array}{c}89,655 \\
(85455-97,305)\end{array}$ & 0.83 & $\begin{array}{c}90,030 \\
(82,725-106,965)\end{array}$ & $\begin{array}{c}84,165 \\
(73,455-105,015)\end{array}$ & 0.04 \\
\hline F Tardía Ins & $\begin{array}{c}27,283 \\
(20,007-42,468)\end{array}$ & $\begin{array}{c}31,284 \\
(22,449-48,237)\end{array}$ & 0.44 & $\begin{array}{c}32,805 \\
(23,250-52,188)\end{array}$ & $\begin{array}{c}26,092 \\
(19,926-40,619)\end{array}$ & 0.34 \\
\hline \multicolumn{7}{|l|}{ CTOG } \\
\hline Glu (0-300') & $89.9 \pm 12.2$ & $91.3 \pm 9.0$ & 0.74 & $88.9 \pm 14.7$ & $99.8 \pm 8.3$ & 0.31 \\
\hline Ins (0-300') & $11.0(7.3-20.0)$ & $11.5(9.8-16.8)$ & 0.31 & $11.3(9.1-16.7)$ & $\begin{array}{c}13.1 \\
(10.8-24.1)\end{array}$ & 0.12 \\
\hline F Temp Glu & $95.5 \pm 11.6$ & $98.1 \pm 10.1$ & 0.54 & $93.4 \pm 4.9$ & $99.8 \pm 8.3$ & 0.02 \\
\hline F Temp Ins & $10.5(7.3-16.7)$ & $12.9(11.3-15.9)$ & 0.18 & $\begin{array}{c}13.8 \\
(10.8-31.9)\end{array}$ & $11.3(6.2-19.1)$ & 0.05 \\
\hline F Tardía Glu & $81.5 \pm 14.0$ & $81.1 \pm 8.6$ & 0.91 & $87.6 \pm 11.3$ & $82.0 \pm 15.6$ & 0.02 \\
\hline F Tardía Ins & $12.1(6.5-19.8)$ & $13.2(7.2-23.5)$ & 0.59 & $12.7(7.1-20.0)$ & $14.0(9.4-16.4)$ & 0.42 \\
\hline \multicolumn{7}{|l|}{ Pico (min) } \\
\hline Mediana & $30(30-60)$ & $30(30-60)$ & 0.61 & $30(27.5-45.0)$ & $30(30-60)$ & 0.41 \\
\hline Promedio & $43.4 \pm 26.5$ & $43.1 \pm 22.2$ & 0.88 & $35.7 \pm 13.9$ & $43.0 \pm 20.0$ & 0.04 \\
\hline \multicolumn{7}{|l|}{ Pico $(\mu \mathrm{U} / \mathrm{ml})$} \\
\hline Mediana & $\begin{array}{c}69.1 \\
(51.4-110.0)\end{array}$ & $\begin{array}{c}77.5 \\
(48.2-107.5)\end{array}$ & 0.69 & $\begin{array}{c}72.7 \\
(52.1-89.5)\end{array}$ & $\begin{array}{c}78.8 \\
(47.4-185.0)\end{array}$ & 0.64 \\
\hline
\end{tabular}

Datos expresados como mediana (intervalo intercuartilar).

AUC: área bajo la curva; CTOG: curva de tolerancia oral a la glucosa; F: fase; Glu: glucosa (mg/dl); Ins: insulina ( $\mu \mathrm{U} / \mathrm{ml})$; Pico: momento (minutos) durante la curva con el valor más alto de insulina ( $\mu \mathrm{U} / \mathrm{ml})$; Tardía: 60-300 min; Temp: temprana (0-30 min).

en el grupo control (11.3 [6.2-19.1]; $p=0.05)$. Estos resultados se resumen en la tabla 2 .

En el momento basal, el pico de insulina en el grupo de sitagliptina ocurrió a los $43.4 \pm 26.5$ minutos, con un valor de 69.1 (51.4-110.0) $\mu \mathrm{U} / \mathrm{ml}$. Este pico de secreción se adelantó a $35.7 \pm 13.9$ minutos después de 2 semanas de tratamiento $(p=0.04)$, con un valor de insulina semejante antes (69.1 [51.4-110.0] $\mu \mathrm{U} / \mathrm{ml})$ y después $(72.7$ [52.1-89.5] $\mu \mathrm{U} / \mathrm{ml} ; p=0.70)$. Por otro lado, el momento de pico de secreción de insulina en el grupo control no se modificó antes en comparación con 2 semanas después de la administración de placebo $(43.1 \pm 22.2$ vs. $43.0 \pm 20.0 \mathrm{~min} ; \mathrm{p}=0.78)$. En el grupo control, el nivel pico de la mediana (intervalo intercuartilar) de insulina también fue semejante al inicio (77.5 [48.2-107.5]) y al final (78.8 [47.4185.0]; $p=0.33$ ) del seguimiento (Tabla 2).

El adelanto de la secreción de insulina en la fase temprana estuvo asociado con un mayor valor del AUC de la glucosa en la fase tardía (minutos 60 a 300) de la CTOG en el grupo con sitagliptina $(90,030[82,725-$ $106,965])$ al compararla con la del grupo control $(84,165$ $[73,455-105,015] ; p=0.04)$. El valor del AUC de la insulina en la fase tardía fue semejante en el grupo de 
sitagliptina $(32,805[23,250-52,188])$ y en el grupo control (26,092 [19926-40619]; $p=0.34$ ) (Tabla 2). Estos resultados también se describen en la figura 2.

\section{Efecto de la sitagliptina en la sintomatología}

En el momento basal, tanto el grupo control como el grupo con sitagliptina presentaron una intensidad de los síntomas semejante (6 [4.0-7.0] y 5 [1.5-6.0], respectivamente; $p=0.12$ ). Después de 2 semanas de tratamiento, el grupo con placebo no mostró cambio significativo en la intensidad de los síntomas (6 [4.0-7.0] vs. 5 [2.0-6.5]; $p=0.23$ ). En contraste la intensidad de los síntomas fue significativamente menor en el grupo con sitagliptina (5 [1.5-6.0] vs. 2.0 [1.0-4.0]; $p=0.01$ ). Al final del seguimiento, la intensidad de los síntomas fue significativamente menor en el grupo con sitagliptina en comparación con el grupo que recibió placebo (2 [2-4] vs. 5 [3.2-6.0]; $p<0.0001)$. En el grupo con placebo se encontró una reducción significativa $(p<0.05)$ en la intensidad de temblor, palpitaciones y diaforesis. En el grupo con sitagliptina hubo una reducción significativa en ansiedad, palpitaciones, irritabilidad, temblor, diaforesis, mareo, hormigueo, dificultad en la concentración y debilidad (todos con $p<0.05$ ) (Tabla 3 ).

\section{Conclusiones}

El fosfato de sitagliptina (100 mg/dl) ocasionó mejoría en la fase temprana de secreción de insulina, lo cual se reflejó en concentraciones mayores de glucosa en la fase tardía de la CTOG. Este cambio biológico tuvo impacto clínico, reduciendo la frecuencia de sintomatología posprandial. Tales hallazgos introducen una totalmente nueva arma terapéutica para pacientes con hipoglucemia reactiva asociada a retraso en la secreción temprana de insulina.
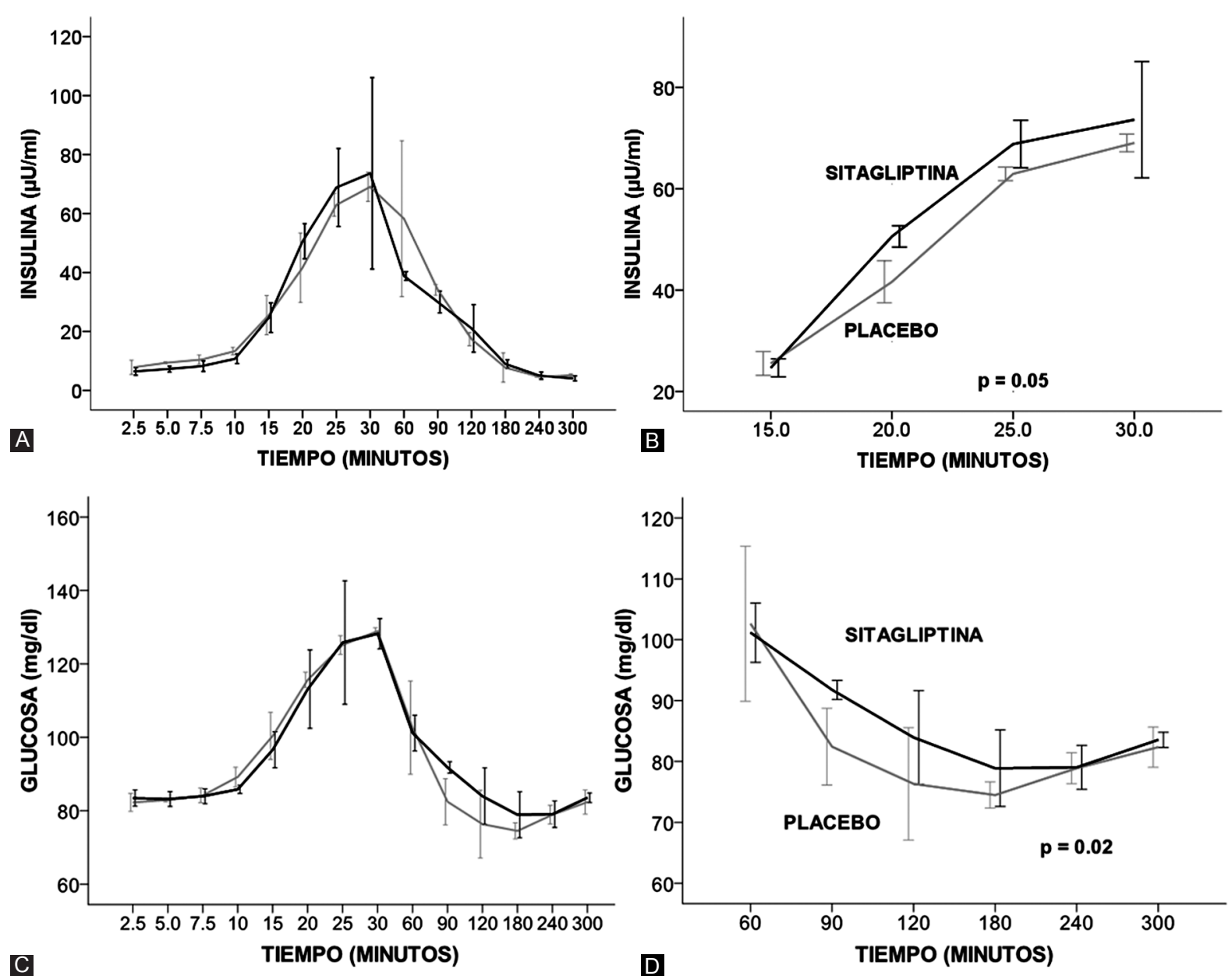

Figura 2. Comparación de los cambios durante la curva de tolerancia oral a la glucosa en la insulina y la glucosa después de recibir sitagliptina (negro) o placebo (gris) en la población estudiada $(n=28)$. Barras de error: intervalos de confianza 95\%. Comparaciones estadísticas mostradas en la tabla 2. 
Tabla 3. Intensidad de los síntomas antes y 2 semanas después de recibir tratamiento con sitagliptina o placebo en la muestra estudiada $(n=28)$

\begin{tabular}{|c|c|c|c|c|c|c|}
\hline \multirow[t]{2}{*}{ Síntoma } & \multicolumn{2}{|c|}{ Sitagliptina } & \multirow[t]{2}{*}{$p$} & \multicolumn{2}{|c|}{ Placebo } & \multirow[t]{2}{*}{$p$} \\
\hline & Antes & Después & & Antes & Después & \\
\hline Todos & $\begin{array}{c}5 \\
(1.5-6.0)\end{array}$ & $\begin{array}{c}2.0 \\
(1.0-4.0)\end{array}$ & 0.01 & $\begin{array}{c}6 \\
(4.0-7.0)\end{array}$ & $\begin{array}{c}5 \\
(2.0-6.5)\end{array}$ & 0.23 \\
\hline Ansiedad & $\begin{array}{c}4 \\
(2.5-5.5)\end{array}$ & $1(1-4.5)$ & 0.003 & $6(4-8)$ & $4(1-6)$ & 0.11 \\
\hline Palpitaciones & $3(1-6)$ & $2(1-3)$ & 0.03 & $4(2-6)$ & $2(1-4)$ & 0.05 \\
\hline Irritabilidad & $3(1-6)$ & $2(1.5-4)$ & 0.04 & $5(3-8)$ & $4(3-8)$ & 0.43 \\
\hline Temblor & $2(1-5)$ & $1(1-2.5)$ & 0.02 & $5(3-8)$ & $5(2-7)$ & 0.008 \\
\hline Diaforesis & $2(1-8)$ & $1(1-5)$ & 0.01 & $6(4-8)$ & $3(1-6)$ & 0.01 \\
\hline Hambre & $5(3-9)$ & $5(1.5-7)$ & 0.42 & $6(3-10)$ & $6(5-9)$ & 0.82 \\
\hline Mareo & $5(1-7.5)$ & $1(1-5)$ & 0.02 & $6(3-9)$ & $5(2-7)$ & 0.06 \\
\hline Hormigueo & $2(1-6.5)$ & $1(1-2.5)$ & 0.03 & $3(2-7)$ & $3(1-6)$ & 0.58 \\
\hline Visión borrosa & $4(1-5)$ & $2(1-5)$ & 0.82 & $3(1-5)$ & $2(1-4)$ & 0.58 \\
\hline $\begin{array}{l}\text { Dificultad } \\
\text { concentración }\end{array}$ & $5(2.5)$ & $2(1.5-4.5)$ & 0.01 & $6(3-8)$ & $5(2-8)$ & 0.41 \\
\hline Debilidad & $4(3-7.5)$ & $2(1-3)$ & 0.003 & $7(3-8)$ & $5(2-8)$ & 0.23 \\
\hline Somnolencia & $4(3-6.5)$ & $2(1-5.5)$ & 0.10 & $7(3-9)$ & $7(4-8)$ & 0.21 \\
\hline
\end{tabular}

Datos expresados como mediana (intervalo intercuartilar).

\section{Financiamiento}

\section{Laboratorio Merck-Sharp \& Dome (MSD).}

\section{Bibliografía}

1. Mentlein R. Dipeptidyl-peptidase IV (CD26) - role in the inactivation of regulatory peptides. Regul Pept. 1999;85:9-24.

2. Mentlein R, Gallwitz B, Schmidt WE. Dipeptidyl-peptidase IV hydrolyses gastric inhibitory polypeptide, glucagon-like peptide-1(7-36)amide, peptide histidine methionine and is responsible for their degradation in human serum. Eur J Biochem. 1993;214:829-35.

3. Kieffer TJ, McIntosh $\mathrm{CH}$, Pederson RA. Degradation of glucose dependent insulinotropic polypeptide and truncated glucagon-like peptide 1 in vitro and in vivo by dipeptidyl peptidase IV. Endocrinology. 1995;136:3585-96.

4. Deacon CF, Nauck MA, Meier J, et al. Degradation of endogenous and exogenous gastric inhibitory polypeptide in healthy and in type 2 diabetic subjects as revealed using a new assay for the intact peptide. J Clin Endocrinol Metab. 2000;85:3575-81.

5. Deacon CF, Johnsen AH, Holst JJ. Degradation of glucagon-like peptide- 1 by human plasma in vitro yields an $\mathrm{N}$-terminally truncated peptide that is a major endogenous metabolite in vivo. J Clin Endocrinol Metab. 1995;80:952-7.

6. Deacon CF, Nauck MA, Toft-Nielsen M, et al. Both subcutaneously and intravenously administered glucagon-like peptide 1 are rapidly degraded from the NH2-terminus in type 2-diabetic patients and in healthy subjects. Diabetes. 1995;44:1126-31

7. De Meester I, Durinx C, Bal G, et al. Natural substrates of dipeptidyl peptidase IV. Adv Exp Med Biol. 2000;477:67-87.

8. De Meester I, Lambeir AM, Proost $P$, et al. Dipeptidyl peptidase IV substrates: an update on in vitro peptide hydrolysis by human DPPIV. Adv Exp Med Biol. 2003;524:3-17.

9. Deacon CF. Therapeutic strategies based on glucagon-like peptide 1 . Diabetes. 2004;53:2181-9.
10. Orskov C, Wettegren A, Holst JJ. Biological effects and metabolic rates of glucagon like peptide-1 7-36 amida and glucagon like petide-1 7-37 in healthy subjects are indistinguishable. Diabetes. 1993;42:658-61.

11. Drucker DJ. The biology of incretin hormones. Cell Metab. 2006;3:153-65.

12. Ahren B, Landin-Olsson M, Jansson PA, et al. Inhibition of dipeptidyl peptidase-4 reduces glycemia, sustains insulin levels, and reduces glucagon levels in type 2 diabetes. J Clin Endocrinol Metab. 2004;89:2078-84.

13. Åhren B, Gomis R, Standl E, et al. Twelve- and 52-week efficacy of the dipeptidyl peptidase IV inhibitor LAF237 in metformin-treated patients with type 2 diabetes. Diabetes Care. 2004;27:2874-80.

14. Åhren B, Landin-Olsson M, Jansson PA, et al. Inhibition of dipeptidyl peptidase-4 reduces glycemia, sustains insulin levels, and reduces glucagon levels in type 2 diabetes. J Clin Endocrinol Metab. 2004;89:2078-84.

15. Scott R, Herman G, Zhao P, et al. Twelve-week efficacy and tolerability of MK-0431, a dipeptidyl peptidase IV (DPPIV) inhibitor, in the treatment of type 2 diabetes (T2D). Diabetes 2005;54(Suppl 1): 10-11 (abstr. 41-OR)

16. Hanefeld M, Herman G, Mickel C, et al. Effect of MK-0431, a dipeptidyl peptidase IV (DPP-IV) inhibitor, on glycemic control after 12 weeks in patients with type 2 diabetes. Diabetologia. 2005;48(Suppl 1):287-8 (abstr. 791).

17. Johnson D, Dorr K, Swenson W, et al. Reactive hypoglycemia. JAMA. 1980;243:1151-5

18. Brun JF, Fedou C, Mercier J. Postprandial reactive hypoglycemia. Diabetes Metab. 2000;26:337-51.

19. Service F. Classification of hypoglycemic disorders. Endocrinol Metab Clin North Am. 1999:28:501-17.

20. Freinkel N, Metzger B. Oral glucose tolerance curve and hypoglycemias in the fed state. N Engl J Med. 1969;280:820-8.

21. Hofeldt F. Reactive hypoglycemia. Endocrinol Metab Clin North Am. 1989;18:185-201.

22. Luyckx A, Lefebvre P. Plasma insulin in reactive hypoglycemia. Diabetes. $1971 ; 20: 435-42$

23. Tablas de valor nutritivo de los alimentos de mayor consumo en Latinoamérica. Instituto Nacional de Ciencias Médicas y Nutrición Salvador Zubirán. México: Editorial Pax; 1996.

24. American Diabetes Association. Introduction. Diabetes Care. 2017;40(Suppl 1):S1-S2. 